kidneys. Suppression of malaria toxicity promotes a quicker restoring of an adequate immune response of the body.

This approach of intensive care with the preventive procedure of extracorporeal hemocorrection method led to a reduction in mortality from 84 to $6.8 \%$ in patients with severe forms of $P$. falciparum malaria.

\subsection{7}

doi: 10.15789/2220-7619-2018-4-4.27

\section{CURRENT PROBLEMS IN DIAGNOSTICS AND TREATMENT OF STRONGULOIDIASIS}

A.K. Tokmalayev, N.A. Polovinkina, V.V. Konnov, K. Emerole Peoples' Friendship University (RUDN University), Moscow, Russia

Long-term observations of patients with various helminthiases showed that strongyloidiasis remains one of the most problematic regarding diagnosis of the disease. It is difficult to make a differential diagnosis on the basis of clinical symptoms due to its polymorphism.

Diagnostic errors of strongyloidiasis were discovered in 38 enrolled patients. It should be noted that incorrect diagnosis led to a change in the nosological structure of the disease. Years ago strongyloidiasis was misdiagnosed with such pathological conditions as acute or chronic enteritis, pancreatitis, bile duct dyskinesia, eosinophilic pneumonia, food poisoning, food infection, typhoid paratyphoid and nontyphoid disease. In recent years, strongyloidiasis was taken for acute leukemia, malignant tumors, Whipple's syndrome and Crohn's disease. Other investigators (N.I. Tumolskaya et al., 2014) reported about "masks" of strongyloidiasis.

Despite a comprehensive approach to laboratory diagnostics of chronic strongyloidiasis according to guidelines, examination of stool specimens (baermann technique) and investigation of the duodenal contents are rarely implemented in clinical laboratories. Therefore parasitological diagnosis of helminthiasis often is established with a significant delay.

Ivermectin is currently the drug of choice in the treatment of strongyloidiasis. It is suitable for the treatment of acute, chronic and disseminated forms of the diseases. A Nobel Prize in 2015 was awarded for its discovery. In the Russian Federation, ivermectin is neither registered nor produced. An alternative drug albendazole is used with a daily dose of 400-800 mg 1-2 times for 3 days. Albendazol is a drug of foreign origin and is available all over the country. Its effectiveness is insufficient and in some cases repeated courses of treatment are required. With early diagnostics and treatment with effective anthelmintic drugs and adequate rehabilitation pathogenetic therapy, the prognosis is usually good, with the exception of immune compromised cases (HIV/AIDS, tuberculosis, non-specific inflammatory diseases, etc.).

\section{ANALYSIS OF POPULATIONS OF BACILLUS ANTRACIS} STRAINS ON THE BASIS OF THEIR RESISTANCE TO SPECIFIC ANTHRAX BACTERIOPHAGES

\section{O.I. Tsygankova, E.A. Koteneva, A.V. Kalinin}

Stavropol Plague Control Research Institute, Stavropol, Russia

The specific anthrax bacteriophage lysis is a compulsory test in the scheme of identification of $B$. anthracis strains, however it does not enable us to estimate quantitatively the presence of phage resistant clones in the population of the strain.

The aim of the study was to investigate the population composition of two virulent strains of $B$. anthracis on the base of phage resistance to specific bacteriophages.
Spore suspensions of typical virulent $B$. anthracis strains 1 (SO) and 81/1 were used as suspensions in a $30 \%$ glycerin solution kept in sealed ampoules at $4-6^{\circ} \mathrm{C}$ for more than 20 years. Concentrations of phage corpuscles in experimental batches of bacteriophages Gamma A-26, BA-9, K-VIEV were, correspondingly, $8 \times 10^{9}, 4 \times 10^{8}$ and $2 \times 10^{8}$ per $1 \mathrm{ml}$. Accurately $0.1 \mathrm{ml}$ of a spore suspension in a concentration of $1 \times 10^{3}$ were applied to Hottinger's agar and spread over its surface. When the liquid was absorbed completely, one of the bacteriophage preparations was applied to test plates, moistening the whole surface of plates. Plates which were not treated with bacteriophage preparations served as controls.

Not a single colony grew on plates of both strains treated with bacteriophage Gamma A-26. Plates treated with bacteriophage K-VIEV showed a $2.9 \%$ growth of colonies of the control of the strain B. anthracis 1 (SO) and a $4.8 \%$ growth of colonies of the strain B. anthracis 81/1. Plates treated with phage BA-9 showed a $10.9 \%$ growth of colonies of the strain $B$. anthracis $1(\mathrm{SO})$ and a $17.3 \%$ growth of colonies of the strain B. anthracis 81/1, correspondingly. For further determination of sensitivity to all the three bacteriophages we used 12 colonies of each strain, which showed resistance to phages K-VIEV and BA-9 at the first stage.

The retest showed that in both strains $16.7 \%$ of variants separated on the base of their resistance to bacteriophage BA-9 were sensitive to all the three bacteriophages. Among variants of the strain $B$. anthracis $81 / 1$ which were selected from the plates treated with bacteriophage BA-9 such variants made up $20 \%$, and in B. anthracis 1 (SO) - 10\%. Among variants of both strains variants resistant to the action of bacteriophages Gamma A-26 and BA-9 and sensitive to bacteriophage K-VIEV were found. Among variants of $B$. anthracis 1 (SO) selected from cultures treated with bacteriophage BA-9, $80 \%$ were sensitive to bacteriophages BA- 9 and Gamma A-26 and resistant to bacteriophage K-VIEV.

4.29 doi: 10.15789/2220-7619-2018-4-4.29

\section{COMPARATIVE CHARACTERIZATION OF SUBCULTURES ISOLATED FROM A POPULATION OF BACILLUS ANTHRACIS 1 (SO) STRAIN ON THE BASIS OF PHAGE RESISTANCE TO SOME SPECIFIC ANTHRAX BACTERIOPHAGES}

O.I. Tsygankova, E.A. Koteneva, A.V. Kalinin

Stavropol Plague Control Research Institute, Stavropol, Russia

On the basis of many phenotypic properties the causative agent of the anthrax shows not only intraspecific variability between strains, but also intrapopulation variability in some strains.

The aim of the work was to study a complex of phenotypic properties and genetic characteristics of variants of the virulent strain $B$. anthracis 1 (SO) in the group of isolated on the basis of resistance to specific anthrax bacteriophages Gamma A-26, BA-9, K-VIEV and to carry out their comparative analysis.

We used the initial strain B. anthracis 1 (SO). Concentrations of phage corpuscles in experimental batches of bacteriophages Gamma A-26, BA-9, K-VIEV were, correspondingly, $8 \times 10^{9}, 4 \times 10^{8}$ and $2 \times 10^{8}$ per $1 \mathrm{ml}$. The criterion for selection of cultural variants was their resistance to bacteriophages. Phenotypic properties and genetic characteristics of isolated subcultures were defined according to the Guidelines 4.2.2413-08.

After treatment of spore cultures on Hottinger's agar with each of the bacteriophages separately, incubation for 24 hours at $37^{\circ} \mathrm{C}$, phage resistant cultural variants being distinguished from the initial typical strain by capsule formation and toxin production, hemolytic, proteolytic 